\title{
Minimizing Bitline Coupling Noise in DRAM with Capacitor-Equiplanar-to-Bitline (CEB) Cell Structure
}

\author{
Li-Fu Chang*, Yu-Ming Hsu, and Min-hwa Chi \\ Technology Development, Vanguard International Semiconductor Cor., \\ *Tel: (886)-3-577-0355, Fax: (886)-3-578-3652, lfchang@mail.vis.com.tw \\ Hsinchu, Taiwan 30077, Republic of China
}

\begin{abstract}
A new scheme of stack DRAM referred to as Capacitor-Equiplanar-to-Bitline (CEB) is proposed for minimizing bitline coupling noises. The cell capacitors are fabricated in between bit-lines, so that the bit-line coupling is blocked by the node capacitor and shielded by the plate. 3D simulation shows that the bit-line coupling noise is almost eliminated ( $<1 \%$ of total bit-line capacitance) in CEB scleme. The SPICE simulation shows $\sim 3 \mathrm{~ns}$ faster bit-line signal sensing in $0.25 \mu \mathrm{m} 64 \mathrm{Mb}$ CMOS DRAM. The CEB scheme also leads to smaller topology and simpler fabrication process.
\end{abstract}

\section{Introduction}

A typical stack DRAM cell [1] is composed of one n-MOS pass transistor and one stack capacitor for storing charges. In order to maintain large enough signal-to-noise ( $\mathrm{S} / \mathrm{N}$ ) ratio, the cell capacitance needs to be $\sim 30 \mathrm{fF}$. The stack capacitor is typically fabricated before bit-line formation in $4 \mathrm{Mb}$ DRAM or earlier and is known as Capacitor-Under-Bitline (CUB) scheme. As DRAM scaling continues, the CUB scheme becomes increasingly difficult for maintaining large enough capacitance. In 16Mb DRAM or newer generations [2], the capacitor is commonly fabricated after the formation of bit-line, as known as Capacitor-Over-Bitline (COB) scheme. In COB scheme, the 
node capacitor can be high and can utilize the space above bit-line for larger capacitance. Both CUB and $\mathrm{COB}$ schemes result in large capacitor topology, which causes process difficulties (e.g. high aspec ratio contacts, planarization, ...etc.). Furthermore, both CUB and COB schemes suffer bit-line coupling noise, which is one of the most serious problems for future DRAMs [3-6].

\section{D Structure of $8 F^{2}$ Cell}

The $8 F^{2}$ cell [7] with either $\mathrm{COB}$ or CUB scheme can not enlarge the capacitor footprint by taking advantage of the space over or under bit-lines as limited by the lithography resolution (i.e. $F$ ). A 3D structure of $8 F^{2}$ DRAM cell with crown-like capacitor has been developed for simulation. The cross-section of the cell structure with COB, CEB, and CUB is sketched in Fig.1. The bit-line and crown capacitor are connected through poly plugs with height of $\mathrm{C}_{1 \mathrm{~h}}$ and $\mathrm{C}_{2 \mathrm{~h}}$ respectively. By varying $C_{l h}$ (or the bitline-to-plate distance $d$ in Fig.1), the bit-line coupling capacitance of various schemes can be simulated [9] using reflective boundary conditions on the 3D capacitance solver Raphacl RC3 (Version 4.0). The cell size is $4 F \times 2 F\left(=8 F^{2}\right)$ with a crown-like capacitor of $\sim 30 \mathrm{fF}$. The bit-line junction capacitance is not included in this simulation.

\section{Bit-line Coupling Capacitance Simulation}

The 3D simulated bitline-to-bitline coupling capacitance $C_{b l b l}$ of $C O B, C E B$, and CUB schemes is shown in Fig.2a. Clearly, the CEB scheme results in minimal $C_{b l b l}$, a result from the blocking of electric field lines by the node capacitor and the shielding by the plate. This would minimize bit-line coupling capacitance to a negligible level, although the bit-line to plate capacitance increases. There is no capacitor blocking in $\mathrm{COB}$ or $\mathrm{CUB}$ schemes, therefore, the bit-line coupling capacitance is larger as shown in Fig.2a. The total bit-line capacitance (not including junction capacitance) as well as various capacitance components are shown in Fig.2b, where the CEB scheme results in the lowest coupling but higher total bit-line capacitance. When the bit-line is closer to the metal layer in CUB scheme, the total bit-line capacitance increases due to metal shielding effect (i.e. termination of field lines from bit-line to metal). 


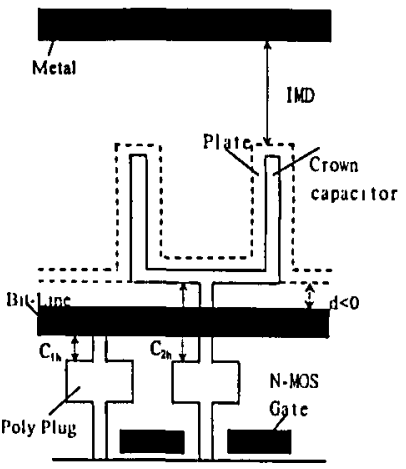

(a) $\mathrm{COB}$

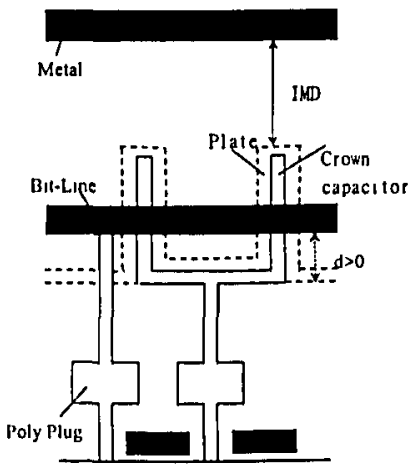

(b) $\mathrm{CEB}$

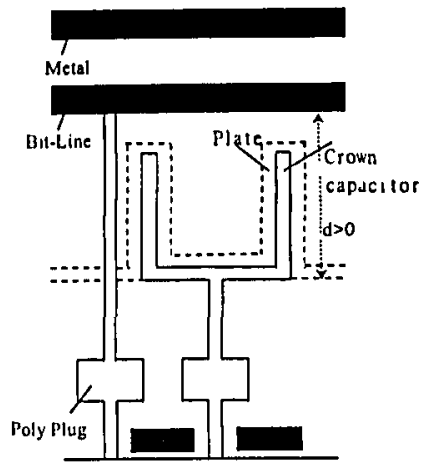

(c) CUB

Fig1: Cross-section of 3D structure of $8 F^{2}$ DRAM cell with crown-like capacitor in $C O B(a), C E B$ (b), and CUB (c) schemes.

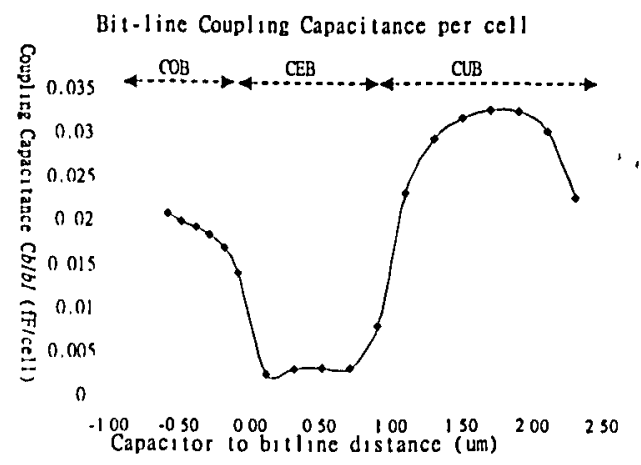

(a)

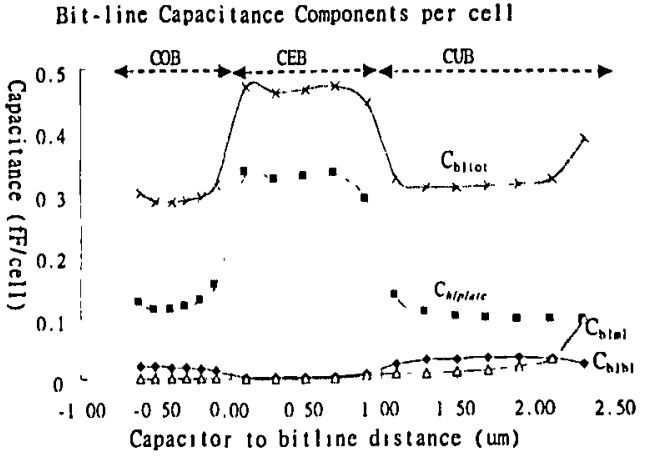

(b)

Fig.2. 3D simulated bitline coupling capacitance $C_{b l b l}$ (a) and various components of cell (b) in $\mathrm{COB}, \mathrm{CEB}$, and CUB schemes. The total bit-line capacitance does not include junction capacitance.

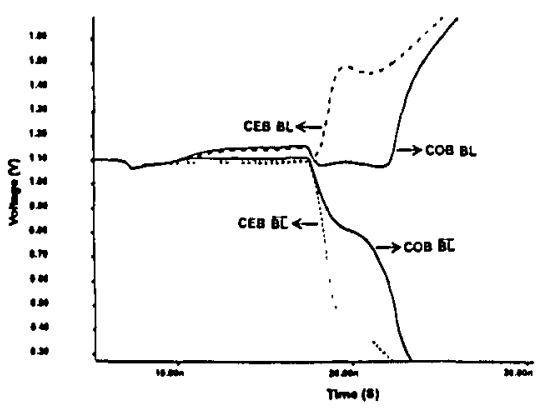

(a)

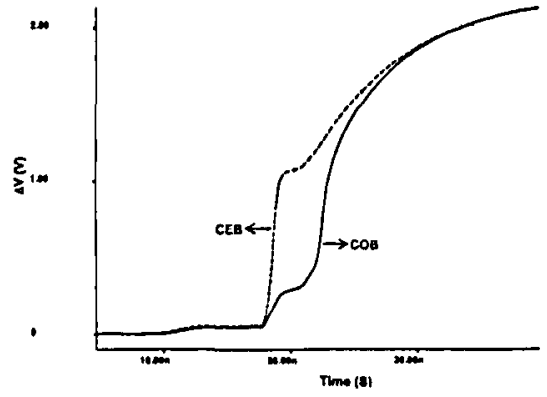

(b)

Fig.3: The bit-line pair potentials (a) after sense amplifier activation and differential bit-line signal (b) in CEB and COB schemes for comparison. 


\section{Bit-line Signal Simulation}

A SPICE simulation of array with COB and CEB schemes is shown in Fig. 3 with 256 cells in a bit-line and including both inter- and intra-bitline couplings. Cell and transistor models are calibrated on a stacked $0.25 \mu \mathrm{m}$ CMOS DRAM technology. Fig.3a shows the bit-line pair potentials after activating sense amplifier are separated faster by $\sim 3 \mathrm{~ns}$ with the new CEB scheme than $C O B$ scheme. Fig.3b shows the differential bit-line signal in $\mathrm{CEB}$ and $\mathrm{COB}$ scheme for comparison.

\section{Conclusions}

In this paper we propose a new $C E B$ scheme for minimizing bitline coupling noises. 3D simulation shows that the bit-line coupling capacitance in CEB is almost eliminated by capacitor blocking and plate shielding. The SPICE simulation shows that CEB results in $>3$ ns faster bit-line signal sensing than other schemes. The CEB scheme leads to smaller capacitor topology and simpler fabrication process significantly.

\section{References}

[1] B. Ei-Kareh, et.al., Solid-State Technology, p.89, 1997.

[2] K. Itoh, et. al., IEEE J. of Solid-State Circuits, V.32, No.5, p.624-634, 1997.

[3] D. Min and D. W. Langer, IEE Electronics Letters, V.33, No.16, p.1380, 1997.

[4] H.Hidaka, et.al., IEEE J. of Solid-State Circuits, V.24, No.1, pp.21-27, 1989.

[5] Y. Konishi, M. Kumanoya, H. Yamasaki, K. Dosaka, and T. Yoshihara, IEEE J. of Solid-State Circuits, V.24, No.1, p.35-42, 1989.

[6] Y. Nakagome, et. al., IEEE J. of Solid-State Circuits, V.23, No.5, p.1120-1127, 1988.

[7] D. Takashima, et. al., IEEE J. Solid-State Circuits, V.29, No.4, p.539-542, 1994.

[8] Y.Kohyama, et.al., Digest of Technical Papers, Symp. on VLSI Technology, pp.17-18, 1997.

[9] D. H. Cho, et.al, IEDM Conference Proceedings, p. 23.2.1 - 23.2.4, December 1996. 